\title{
Comparative Dissociation of Peptide Polyanions by Electron Impact and Photo-Induced Electron Detachment
}

\author{
Vincent Larraillet, ${ }^{\text {a }}$ Aleksey Vorobyev, ${ }^{\mathrm{b}}$ Claire Brunet, ${ }^{\mathrm{a}}$ Jérôme Lemoine, ${ }^{\mathrm{c}}$ \\ Yury O. Tsybin, ${ }^{\mathrm{b}}$ Rodolphe Antoine, ${ }^{\mathrm{a}}$ and Philippe Dugourd ${ }^{\mathrm{a}}$ \\ ${ }^{a}$ Université de Lyon, F-69622, LASIM, Lyon, France \\ b Biomolecular Mass Spectrometry Laboratory, Ecole Polytechnique Fédérale de Lausanne, Lausanne, Switzerland \\ ${ }^{\mathrm{c}}$ Université de Lyon, F-69622, Sciences Analytiques, Lyon, France
}

\begin{abstract}
We compare product-ion mass spectra produced by electron detachment dissociation (EDD) and electron photodetachment dissociation (EPD) of multi-deprotonated peptides on a Fourier transform and a linear ion trap mass spectrometer, respectively. Both methods, EDD and EPD, involve the electron emission-induced formation of a radical oxidized species from a multi-deprotonated precursor peptide. Product-ion mass spectra display mainly fragment ions resulting from backbone cleavages of $\mathrm{C}_{\alpha}-\mathrm{C}$ bond ruptures yielding $a$ and $x$ ions. Fragment ions originating from $\mathrm{N}-\mathrm{C}_{\alpha}$ backbone bond cleavages are also observed, in particular by EPD. Although EDD and EPD methods involve the generation of a charge-reduced radical anion intermediate by electron emission, the product ion abundance distributions are drastically different. Both processes seem to be triggered by the location and the recombination of radicals (both neutral and cation radicals). Therefore, EPD product ions are predominantly formed near tryptophan and histidine residues, whereas in EDD the negative charge solvation sites on the backbone seem to be the most favorable for the nearby bond dissociation. (J Am Soc Mass Spectrom 2010, 21, 670-680) (c) 2010 Published by Elsevier Inc. on behalf of American Society for Mass Spectrometry
\end{abstract}

$\mathrm{O}$ ne of the main advantages of mass spectrometry for peptide and protein structure analysis is its ability to give valuable information from very low amounts of samples, e.g., primary structure (amino acids sequence), number and location of disulfide bridges, identification, and sometimes characterization of post-translational modifications. To provide this information, various activation techniques are used to fragment peptide or protein ions to yield structurespecific product ions complementary to accurate molecular mass measurements [1-6].

Different methods are available nowadays to excite and fragment biomolecular ions. In low-energy collisionactivated dissociation (CAD) and infrared multiphoton dissociation (IRMPD), the peptide ion is heated in a multi-step process $[7,8]$. The model of the mobile proton can rationalize the observed fragments [9]; the transfer of a proton weakens locally the peptide bond, facilitating its fragmentation. Since the fragmentation occurs after heating and vibrational energy redistribution, structural rearrangements are in competition with fragmentation pathways. In addition and in complement to slow heating methods, reactions of polypeptide

Address reprint requests to Dr. R. Antoine, Laboratoire de Spectrométrie Ionique et Moléculaire, Université de Lyon, Université Lyon 1, Villeurbanne, CNRS, UMR5579, LASIM, F-69622, Lyon, France. E-mail: rantoine@ lasim.univ-lyon1.fr ions with electrons and small radical ions have become a very useful tool for peptide structural analysis. For instance, electron capture dissociation (ECD) $[6,10]$ and electron-transfer dissociation (ETD) [11-13] may initiate bond breaking in peptide and protein polycations presumably faster than energy redistribution over all degrees of freedom. Less than a decade ago, an ionelectron interaction-based fragmentation technique for peptide and protein polyanions termed electron detachment dissociation (EDD) was proposed [14-16]. More recently, an ion-ion interaction-based peptide and protein polyanion fragmentation, termed negative ETD, was achieved with small radical cations [13, 17]. EDD has been proposed for analysis of acidic polypeptides, including peptides with labile modifications, whereas negative ETD has not been substantially explored yet. The ion-electron and ion-ion reactions are different from slow heating methods by the fact that the intermediate fragmenting species are odd-electron ions. The presence of a radical site diminishes the strength of nearby bonds. In particular, for peptide polyanions, preferential backbone cleavages of $\mathrm{C}_{\alpha}-\mathrm{C}$ bonds yielding $a$ and $x$ ions were demonstrated [14, 18].

A new method, namely electron photo-detachment dissociation (EPD), was recently developed for the gas-phase dissociation of peptide and DNA polyanions [19-21]. In this method, the radical anions are obtained
(C) 2010 Published by Elsevier Inc. on behalf of American Society for Mass Spectrometry. $1044-0305 / 10 / \$ 32.00$

doi:10.1016/j.jasms.2010.01.015
Published online January 28, 2010 Received December 8, 2009 Revised January 11, 2010 Accepted January 14, 2010 
by electron photo-detachment from a multiply deprotonated species [22]. The fragmentation of these radical anions after collisional activation in multi-stage mass spectrometry was explored for a number of peptides and proteins. Similar to EDD, the dominance of backbone $\mathrm{C}_{\alpha}-\mathrm{C}$ bond ruptures yielding $a$ and $x$ ions was observed in EPD. Nevertheless, a more detailed understanding of the underlying processes in EDD and EPD is needed to further enhance the efficiency of the methods that remain rather low. A direct comparison of EDD and EPD could provide a further insight into the radical chemistry of these two processes and improve our understanding of their mechanisms.

In the present study, we compare the fragmentation patterns of a panel of peptides by EDD and EPD to explore in more detail similarities and differences between these ion activation and dissociation methods. In particular, the influence of the possible radical site location on the product ion abundance distribution is discussed.

\section{Experimental}

\section{Chemicals}

Free acid Substance $\mathrm{P}$ and caerulein were purchased from Sigma-Aldrich (Saint Quentin Fallavier, France). The wild-type and variants of a transmembrane domain of M2 protein, M2 TMP, were synthesized by fluorenylmethyloxycarbonyl (Fmoc) solid-phase peptide synthesis (Peptide and Protein Synthesis Facility, University of Lausanne, Switzerland). Peptides were used without further purification.

\section{Activated-Electron Photodetachment Dissociation}

Activated-EPD experiments were performed using a modified linear quadrupole ion trap mass spectrometer (LTQ; Thermo Fisher Scientific, San Jose, CA, USA) with extension for the high mass to charge ratio range 2000-4000 Th. Peptides were dissolved in 50/50 water / acetonitrile ( $\mathrm{vol} / \mathrm{vol})$ at a concentration of $100 \mu \mathrm{M}$ and directly electrosprayed at a flow rate of $5 \mu \mathrm{L} / \mathrm{min}$ for subsequent analysis in a negative-ion mode. A quartz window was fitted on the rear of the LTQ chamber to allow the introduction of a UV laser beam. The laser is a nanosecond frequency-doubled tunable Panther EX OPO laser pumped by a Surelite II Nd:YAG laser (both from Continuum, Santa Clara, CA, USA). All the EPD experiments were performed at $\lambda=260 \mathrm{~nm}$. The output power for this wavelength was $\sim 10 \mathrm{~mW}$ at a repetition rate of $10 \mathrm{~Hz}$. The laser beam passes through two diaphragms (2 mm diameter), lenses, and a mechanical shutter electronically synchronized with the mass spectrometer, after which, it is injected on the axis of the linear trap. The mechanical shutter is used to synchronize the laser irradiation with the trapping of the ions. To perform laser irradiation for a given number of laser pulses, we complemented the ion trap rf sequence with a $\mathrm{MS}^{n}$ step with activation amplitude of $0 \%$, during which the shutter located on the laser beam is opened. The maximum laser energy that enters the trap is $\sim 600$ $\mu \mathrm{J} /$ pulse. Collision activated dissociation (CAD) experiments were performed with the same apparatus using helium gas. Activation time for CAD was $30 \mathrm{~ms}$ and collision energy was between $15 \%$ and $20 \%$. For activatedEPD experiments, the oxidized species is isolated in a $\mathrm{MS}^{3}$ step and further activated by collisions. Activation time for the $\mathrm{MS}^{3} / \mathrm{CAD}$ step was $30 \mathrm{~ms}$ and collision energy was between $15 \%$ and $20 \%$. For both CAD and activated-EPD experiments, activation value $\mathrm{q}$ was set to 0.25 and a $m / z$ window of 5 Th was applied for precursor ion isolation.

\section{Electron Detachment Dissociation}

A hybrid 7-T linear ion-trap Fourier transform (LTQ FT) ion cyclotron resonance mass spectrometer (Thermo Fisher Scientific, Bremen, Germany) equipped with an indirectly heated dispenser cathode as an electron source was used for the EDD experiments. Peptides were dissolved in 50/50 water/acetonitrile (vol/vol) at a concentration of $2 \mu \mathrm{M}$. Negative ions of interest were produced in a regular microspray ESI source with a liquid flow rate of $10 \mu \mathrm{L} / \mathrm{min}$, isolated with a window of $5 \mathrm{Th}$ and accumulated in the LTQ (AGC setting of $500,000)$ for further transfer to the ICR cell. The delay period between ion injection into the ICR cell and irradiation with an electron beam was tuned to account for ion magnetron motion [23]. The electron energy of $\sim 10 \mathrm{eV}$, estimated for employed ECD electron energy parameter (Xcalibur, Thermo Fisher Scientific) of about -13 to $-16 \mathrm{~V}$ and ICR cell trapping potentials of $\sim 1 \mathrm{~V}$ and interaction time of $\sim 1 \mathrm{~s}$ were selected to obtain maximum signal to noise ratio for EDD fragments for free acid Substance P employed as a reference peptide. The optimized parameters were then used for all other samples. The tandem mass spectra were obtained at 50,000 resolution at $\mathrm{m} / \mathrm{z} 400$ and were produced by averaging of 200 microscans.

\section{Methods}

Both electron detachment dissociation (EDD) and electron photodetachment dissociation (EPD) are initiated by inducing electron emission from peptide polyanion that results in formation of a radical oxidized species and its consequent decomposition with or without supplemental vibrational activation. The principles and the mechanistic aspects of these two dissociation methods are described in more details in this section.

\section{Dissociation by Electron Photodetachment with Supplemental Ion Activation (Activated-EPD)}

As illustrated by Scheme $\mathbf{1}$ below, the EPD method consists of an initial step of UV irradiation (around 4.6 


\section{$[M-n H]^{n-} \stackrel{h v}{\longrightarrow}[M-n H]^{(n-1)-\bullet}+e^{-}$ $\stackrel{C A D}{\longrightarrow}$ peptide fragment ions}

Scheme 1

$\mathrm{eV}$ per photon) of a multiply deprotonated peptide. The main consequence of UV irradiation of such species is the loss of one electron resulting in an oxidized form of the precursor ion.

For peptides that contain natural aromatic aminoacids (i.e., tryptophan, tyrosine, and, in a lesser extent, phenylalanine), absorption at $\sim 260 \mathrm{~nm}$ is mainly due to a resonant electronic excitation ( $\pi-\pi *$ transition) [22, 24]. The electronic excitation leads to the electron loss that occurs after electron relaxation. The excess energy let in the oxidized form of the precursor ion [given by the difference between electron binding energy $(\sim 2.5-$ $3.5 \mathrm{eV})[22,24]$ and the photon energy $(\sim 4.6 \mathrm{eV})]$ is distributed between the electron kinetic energy and the internal energy of the radical ion. This results in a radical with low excess internal energy. The oxidized species is isolated and further activated by collisions. In the case of peptides, the subsequent CAD of the isolated radical anions results in intense backbone cleavages. EPD fragmentation patterns are characterized, for small peptides, by dominant backbone cleavage of $\mathrm{C}_{\alpha}-\mathrm{C}$ bonds giving $a$ and $x$-type product ions [20]. For larger peptides, fragment ions originating from $\mathrm{N}-\mathrm{C}_{\alpha}$ and $\mathrm{C}_{\alpha}-\mathrm{C}$ backbone cleavages are also observed [21].

\section{Dissociation by Electron Detachment (EDD)}

As illustrated by Scheme 2 below, EDD utilizes fast, $>10 \mathrm{eV}$, electrons to detach electrons from multiply deprotonated peptides by electron impact. The proposed mechanism involves the polypeptide chain ionization leading to the creation of a positive radical charge (hole). Mutual neutralization of the hole and the electron results in electronic excitation that causes fragmentation. The recombination of the electron-hole pair is exothermic and is approximately equal to the difference between the ionization energy of a polypeptide chain and the electron affinity of the negatively charged group (usually carboxylate groups), i.e., $\sim 5 \mathrm{eV}$ [15]. Spontaneous fragmentation occurs and additional vibrational energy is not necessary.

Similar to EPD, EDD fragmentation is characterized by dominant backbone cleavage of $\mathrm{C}_{\alpha}-\mathrm{C}$ bonds giving $a$ and $x$-type product ions [14]. The characteristic fragmentation patterns of both EPD and EDD allow for efficient peptide sequencing and stand in sharp contrast to the nonspecific fragmentation obtained with CAD in the negative ion mode. However, EDD is a rather inefficient process [25] due to its limited reaction cross section between negatively charged polypeptides and electrons of the required energy.

\section{Results and Discussion}

This report is specifically focused on a comparison of the product ion mass spectra using EDD on a FTMS instrument and EPD on a linear ion trap mass spectrometer. The influence of the charge location, the charge state, and the nature of chromophore on the product ion pattern is explored across peptide models with molecular mass ranging from 1300 to $2800 \mathrm{Da}$.

\section{EPD and EDD of Substance P-OH}

EDD of the dianions $[\mathrm{M}-2 \mathrm{H}]^{2-}$ of the free acid $1.4 \mathrm{kDa}$ peptide Substance $\mathrm{P}$ (RPKPQQFFGLM-OH) is shown in Figure 1a. A series of $x$ ions dominate the product ion mass spectrum, with $x_{7}$ being the most abundant. An extensive $\mathrm{CO}_{2}$ loss is also observed. The present mass spectrum is very similar to the one obtained in the Paul ion trap mass spectrometer and previously reported by Zubarev and coworkers [15]. The $\mathrm{CO}_{2}$ loss results from the facile decarboxylation of the deprotonated C-terminal carboxylic acid group after irradiation-catalyzed oxidation by energetic electrons [15]. The dominating $x$ ion series is in agreement with preferential charge retention by the C-terminal carboxylic acid. Some $a$ ions are observed with a very low intensity compared with $x$ ions.

The described similarity in product ion mass spectra between EDD in high vacuum FT-ICR MS and in much lower vacuum of the ion trap MS is important in view of the different experimental conditions, in particular precursor ion internal energy values [26]. In contrast to ECD and ETD comparison described by Ben-Hamidane et al. [26], energy of impacting electron is substantially higher in EDD, thus additional activation is not required for the decomposition of noncovalently bound EDD products even in the ion trap MS conditions. In case of EPD in ion trap MS, the activation step is important, similarly to ETD, as described in the Methods section. Based on the comments above, we assume that comparative analysis of total product ion mass spectra, represented by summed up even- and oddelectron components, of EPD and EDD described here is justified. Nevertheless, a direct comparison of EPD in ion trap MS and FT-ICR MS is required to conclude on the impact of experimental parameters, e.g., vacuum, on product ion mass spectra in general and on the associated radical-ion involved hydrogen atom rearrangements processes in particular.

The EPD spectrum of the acidic form of Substance P is dominated by neutral losses (see Figure 1b). Although not the dominant neutral loss, the $\mathrm{CO}_{2}$ loss is

$$
\begin{aligned}
& {[M-n H]^{n-}+e_{\overline{h o t}}^{-} \rightarrow[M-n H]^{(n-1)-\bullet *}+2 e^{-}} \\
& \rightarrow \text { peptide fragment ions }
\end{aligned}
$$

Scheme 2 


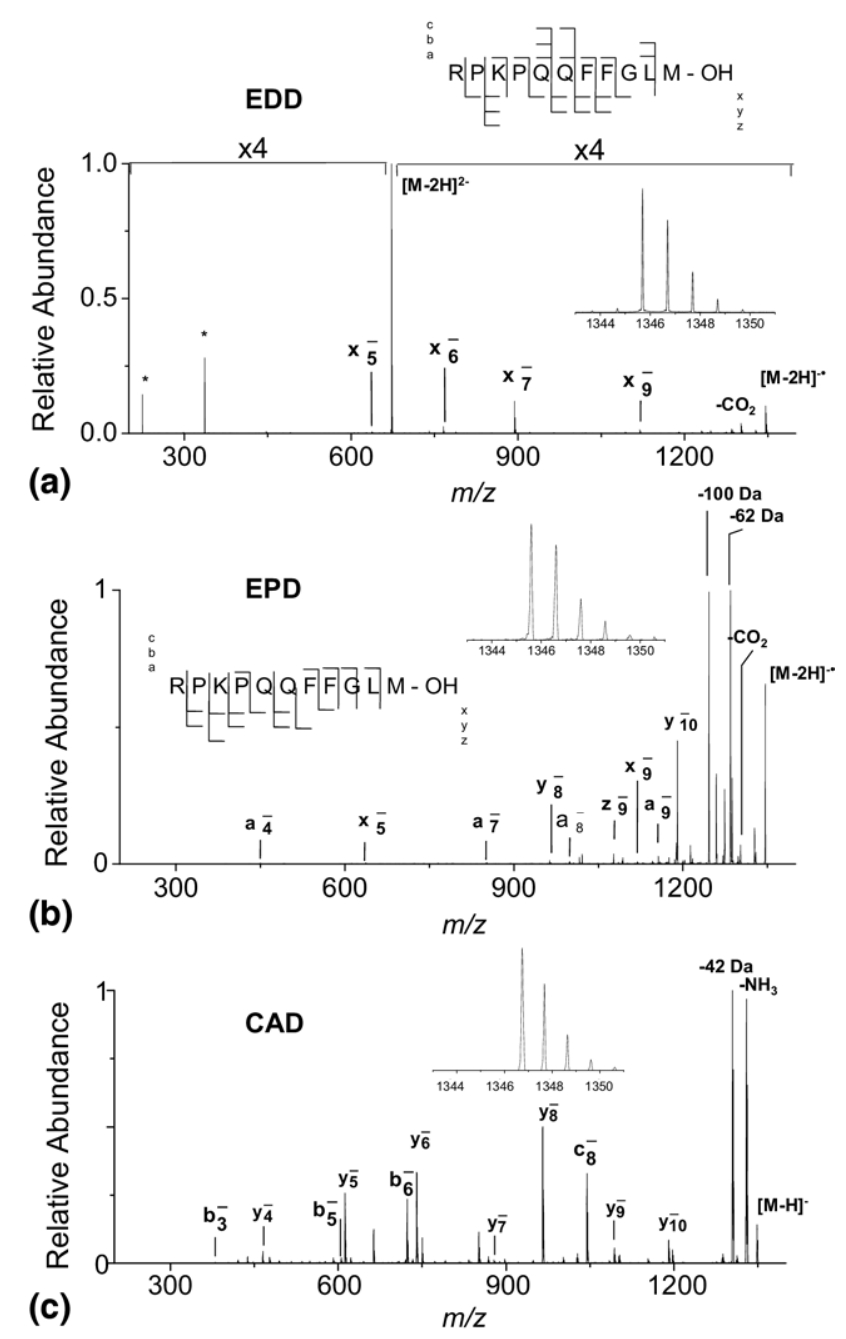

Figure 1. Tandem mass spectrometry of the acidic form of doubly deprotonated Substance P (RPKPQQFFGLM-OH), (a) EDD FT-ICR mass spectrum; double and triple harmonics are shown with asterisk. (b) EPD LTQ mass spectrum. The insets show the isotopic distributions of the corresponding $[\mathrm{M}-2 \mathrm{H}]^{-}$ ions. Activation time for the $\mathrm{MS}^{3} / \mathrm{CAD}$ step was $30 \mathrm{~ms}$ and collision energy was $17 \%$. Only the most intense fragments have been annotated on the spectra. The insets show the sequence of the peptide with all the fragments detected. (c) CAD LTQ mass spectrum. Activation time was $30 \mathrm{~ms}$ and collision energy was $19 \%$.

present in the spectrum and can be related to the oxidation of the deprotonated carboxylic acid. Abundant fragment ions corresponding mainly to losses of 100 and $62 \mathrm{Da}$ are observed and may be a fingerprint of the second deprotonation site. These neutral losses are not observed by collision activation of the even-electron species $[\mathrm{M}-\mathrm{H}]^{-}$(see Figure 1c). CAD experiments performed on these two abundant fragment ions (M100 and M-62 ions) permit to locate the position of the side-chain loss in the peptide sequence. CAD fragmentation data show that losses of 100 and 62 Da correspond, respectively, to side-chain cleavages of the arginine and methionine residues. The loss of $100 \mathrm{Da}$ could be a consequence of a radical formation and recombination on the terminal amine, which is a good candidate as second deprotonation site (as depicted in Scheme 3) $[27,28]$.

The fragment ion corresponding to the loss of $62 \mathrm{Da}$ is attributed to a $\mathrm{CH}_{3}-\mathrm{S}-\mathrm{CH}_{3}$ side-chain loss from the methionine residue in the vicinity of the C-terminal carboxylic group. Note that both the losses of 62 and $100 \mathrm{Da}$ are also observed by EDD, but with a weaker intensity. This specific neutral side-chain loss at the $\mathrm{N}$-terminal side of the peptide (e.g., loss of $100 \mathrm{Da}$ in Substance P) may be used as a fingerprint of the location of a deprotonation site at the N-terminal amine. To probe this hypothesis, we used another nonacidic peptide (MRFA) for which deprotonation sites are supposed to be the same as for Substance P (i.e., carboxylic C-terminal group and the $\mathrm{N}$-terminal amine). This peptide also possesses an arginine residue but not at the $\mathrm{N}$-terminal position. A negligible loss of $100 \mathrm{Da}$ is observed, but an important loss of $62 \mathrm{Da}$ is observed. The loss of $62 \mathrm{Da}$ is attributed to a $\mathrm{CH}_{3}-\mathrm{S}_{-} \mathrm{CH}_{3}$ sidechain loss from the methionine residue in the vicinity of the N-terminal carboxylic group. This last result confirms that a neutral loss from the $\mathrm{N}$-terminal side of the peptide can be used as a fingerprint of the location of deprotonation site at the N-terminal amine for nonacidic peptides.

An intense $y_{10}$ ion is observed in EPD, whereas this fragment is observed only with a weak intensity by EDD. This fragmentation channel corresponds to the cleavage of the amide bond between the arginine and proline residues. It could also originate from the radical generated on the deprotonated N-terminal amine group mentioned above as a recombination pathway competitive to the 100 Da neutral loss. However, cleavage of N-terminal proline is facile and the $y_{10}$ fragment ion could be observed on the CAD spectrum of the nonradical precursor ion (i.e., $[\mathrm{M}-\mathrm{H}]^{-}$ions). The $y_{10}$ ion is indeed observed by collision activation of the evenelectron species $[\mathrm{M}-\mathrm{H}]^{-}$but with a weak intensity (see Figure 1c). Thus, the cleavage of the amide bond between the arginine and proline residues resulting in the observation of enhanced $y_{10}$ ions in the activated-
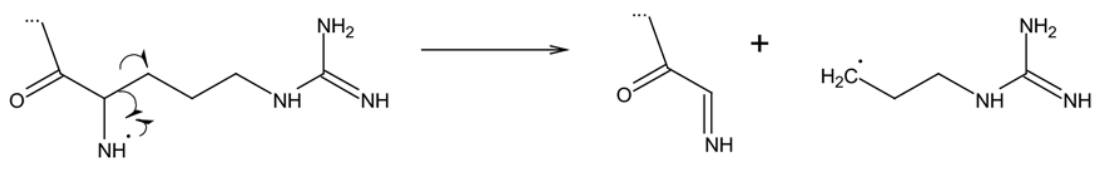

$100 \mathrm{Da}$ 
EPD mass spectrum of substance is certainly influenced by the presence of a radical generated on the deprotonated $\mathrm{N}$-terminal amine group. Observation of $\mathrm{CO}_{2}$ loss on one hand, and of $100 \mathrm{Da}$ loss and enhanced $y_{10}$ ions on the other hand, suggest that the electron loss in EPD could occur both on the carboxylate group and on the deprotonated N-terminal amine. Indeed, in EPD, electron detachment at $260 \mathrm{~nm}$ occurs mainly through a resonant electronic excitation of the peptide, followed by autoionization of the excited peptide. Therefore, the present results suggest that in EPD of Substance P-OH, electron loss may occur from a different deprotonation site compared with the preferential electron loss from the carboxylate group upon electron irradiation in EDD.

Some $x$ ions and a series of $a$ ions are also observed with a significant intensity in EPD of Substance P-OH. Although the $a$ ions are nearly not observed in EDD, these ions are as intense as the $x$ ions in EPD. This observation is consistent with the earlier observation by EDD [14] that the less stable $a$ ions, being radical, are more abundant in QIT mass spectra than in FTMS mass spectra. Presumably, the low-frequency of ion-neutral collisions in FT-ICR MS leads to much longer relaxation times after activation through inelastic collisions with electrons, which results in a higher average temperature of ions during the interval after the EDD event and before ion detection.

\section{EPD and EDD of Caerulein}

The electrospray spectrum of caerulein (pEQDYTGWMDF$\mathrm{NH}_{2}$ ), a sulphated peptide on $\mathrm{Tyr}_{4}$ residue, exhibits an intense $[\mathrm{M}-2 \mathrm{H}]^{2-}$ ion. Five UV laser shots of the isolated dianions give rise to the oxidized radical [M $2 \mathrm{H}]^{--}$species with a yield of $\sim 90 \%$ (see inset of Figure $2 a)$. The high efficiency in electron photodetachment is due to the presence of three chromophores (sulfatedtyrosine, tryptophan, and phenylalanine residues) that strongly absorb laser irradiation suppress of around 260 $\mathrm{nm}$. The radical anion can then be isolated and subjected to collision-induced excitation. The activatedEPD spectrum is shown in Figure 2a. The most prominent losses from the oxidized $[\mathrm{M}-2 \mathrm{H}]^{-\cdot}$ species are $-\mathrm{CO}_{2}$ and $-\mathrm{SO}_{3}$ groups. As already discussed for Substance $\mathrm{P}-\mathrm{OH}$, these neutral losses result from the oxidation of $\mathrm{CO}_{2}{ }^{-}$and $\mathrm{SO}_{3}{ }^{-}$groups. A large peak at $\mathrm{m} / \mathrm{z}$ 1288 is also observed and is attributed to losses of $\mathrm{CO}_{2}$ and $\mathrm{NH}_{3}$ molecules from the oxidized $[\mathrm{M}-2 \mathrm{H}]^{-}$. species. The loss of $\mathrm{NH}_{3}$ is a very common fragmentation feature of Gln-containing peptides such as caerulein [29]. Note also an important loss of $129 \mathrm{Da}(-\mathrm{W})$, which corresponds to a radical neutral loss of the tryptophan side-chain (see Scheme 4a) [30]. Importantly, $a$ - and $c$-type product ion series were observed due to the backbone cleavages (see Figure 2a). Scarce $y$ and $z$ ions were also observed. Most fragment ions

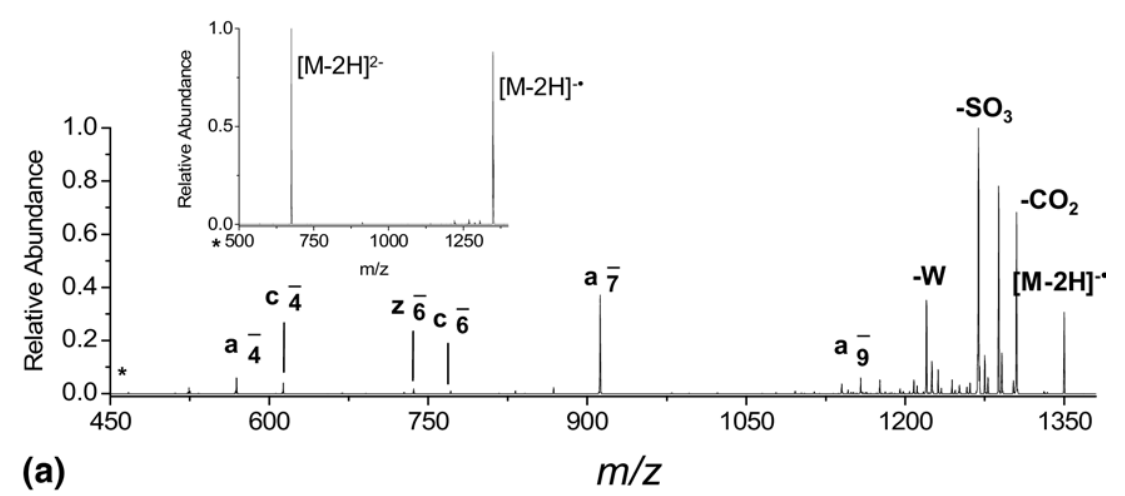

(a)

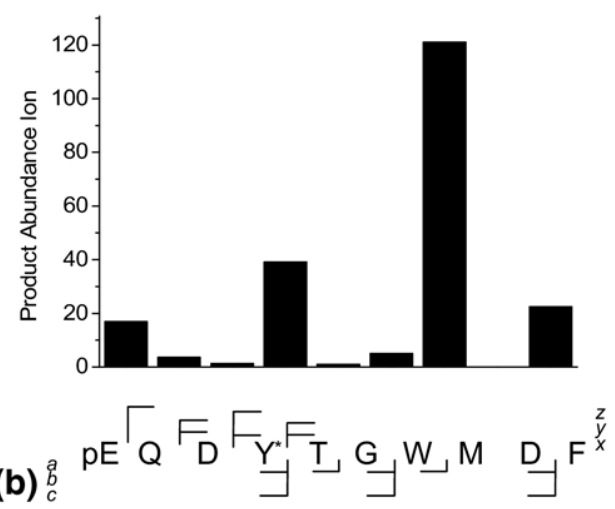

Figure 2. (a) EPD LTQ mass spectra of $[\mathrm{M}-2 \mathrm{H}]^{-\cdot}$ caerulein ions. The insert shows the photodissociation spectrum of isolated $[\mathrm{M}-2 \mathrm{H}]^{2-}$ ions after 5 laser shots at $260 \mathrm{~nm}$. (b) EPD product ion abundance distribution of caerulein (sum of $a$ and $x$ fragment intensities). 
(a)
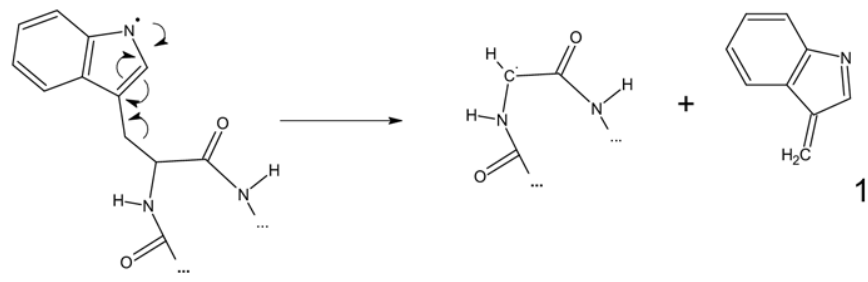

$129 \mathrm{Da}$

(b)
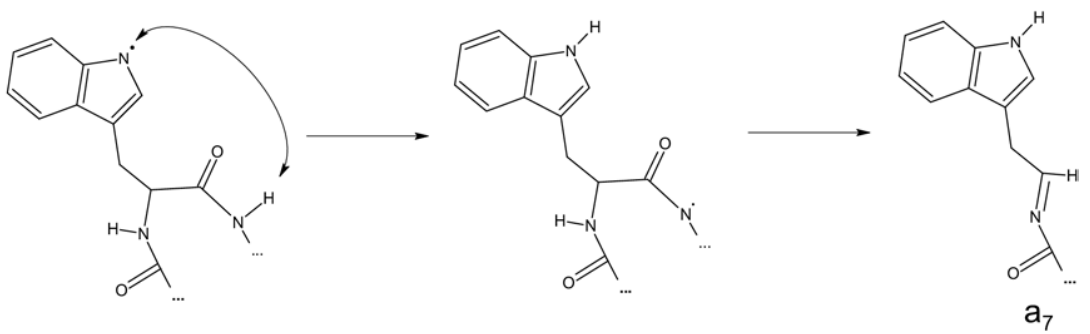

Scheme 4

retained the sulphate group. The $a_{7}$ fragment ion is by far the most abundant and corresponds to the cleavage of the peptide bond between the tryptophan and methionine residue. As already reported by EDD measurements [15], all observed $a$ ions but $a_{7}$ ions were radicals (however, the analysis of the isotopic distribution of $a$ ions shows that minor nonradical species are also present). The loss of $129 \mathrm{Da}$ and the formation of $a_{7}$ ions may be both due to the formation of a neutral Trp radical. The presence of the radical on the nitrogen atom of the indole residue would increase the possibility to form $a$ ions between the tryptophan and methionine residues, as depicted in Scheme $\mathbf{4 b}$.

The most intense fragments discussed above (losses of $\mathrm{CO}_{2}, \mathrm{SO}_{3}, 129 \mathrm{Da}$, and $a_{7}$ fragment ions) are then thought to originate from a charge loss on the $\mathrm{CO}_{2}{ }^{-}$and $\mathrm{SO}_{3}{ }^{-}$followed either by loss of $-\mathrm{CO}_{2}$ and $-\mathrm{SO}_{3}$ molecules or by radical migration to tryptophan.

The caerulein fragmentation pattern observed by EPD contrasts with the previous EDD spectrum reported by Zubarev and coworkers [15]. Indeed, although $a, c$, and $z$ product ion series were observed across the peptide backbone, the most abundant EDD cleavages took place in the vicinity of the sulfated tyrosine residue, one of the two most likely deprotonation sites. Fragments close to the tryptophan residue were found with only low abundance. These differences in the product ion abundance may reflect the fact that radical migration is favored in activated-EPD during the collision activation stage that is not present in EDD. One should note that the chromophore of the Trp side chain acts as an antenna for laser-induced excitation of peptides at $260 \mathrm{~nm}$. Coupling between electronic excited states localized on Trp and autoionization states leading to electron loss from the $\mathrm{CO}_{2}{ }^{-}$and $\mathrm{SO}_{3}{ }^{-}$may be favored for conformations for which Trp and negative charges are relatively close. Initial excitation located on Trp and such favorable conformations may in return induce back radical transfer to Trp.

\section{EPD and EDD of M2 TMP Variants}

To further assess the differences in the fragmentation pattern between EPD and EDD, wild-type transmembrane domain M2 TMP peptide variants, whose sequences are given in Table 1, were studied. Wild-type M2 TMP peptide (M2 TMP wt, SSDPLVVAASIIGILHLILWILDRL-OH) is a transmembrane domain of the membrane protein M2 from influenza virus A [31]. In M2 TMP, L13@G13, Gly 13 $_{13}$ of M2 TMP wt is replaced with a Leu residue, whereas in M2 TMP, G7@V7, the $\mathrm{Val}_{7}$ is replaced by Gly residue. Finally, in M2 TMP, G16@H16, the $\mathrm{His}_{16}$ is replaced by a Gly residue. These variants have already been studied by electron capture dissociation (ECD) and displayed the corresponding modulation of product ion abundance distribution [26, 32].

In the present work, experiments were performed starting with the triply deprotonated $[\mathrm{M}-3 \mathrm{H}]^{3-}$ species. EDD of M2 TMP, G7@V7 peptide, Figure 3a, shows an intense loss of $\mathrm{CO}_{2}$ molecules, as already observed for the acidic form of Substance P. Mainly, series of singly deprotonated $a$ and $x$ product ions are observed due to the backbone cleavages, as summarized in Figure 4a. The fragmentation is particularly efficient near the center of the peptide between the $\mathrm{Ile}_{11}$ and $\mathrm{Ile}_{12}$ residues, where both $a_{11}$ and $x_{14}$ ions are very

Table 1. Names, sequences, and molecular weights of wild-type transmembrane domain M2 TMP peptide and its variants

\begin{tabular}{llc}
\hline \multicolumn{1}{c}{ Peptide } & \multicolumn{1}{c}{ Sequence } & $\begin{array}{c}\text { Molecular } \\
\text { weight, } \\
\text { Da }\end{array}$ \\
\hline \hline M2 TMP, wt & SSDPLVVAASIIGILHLILWILDRL & 2727 \\
M2 TMP, & SSDPLVVAASIILILHLILWILDRL & 2783 \\
L13@G13 & & \\
M2 TMP, G7@V7 & SSDPLVGAASIIGILHLILWILDRL & 2685 \\
M2 TMP, & SSDPLVVAASIIGILGLILWILDRL & 2647 \\
G16@H16 & & \\
\hline
\end{tabular}




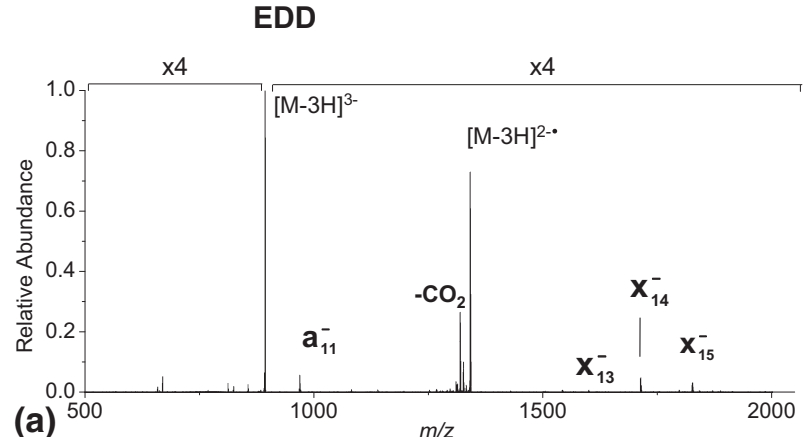

(a)

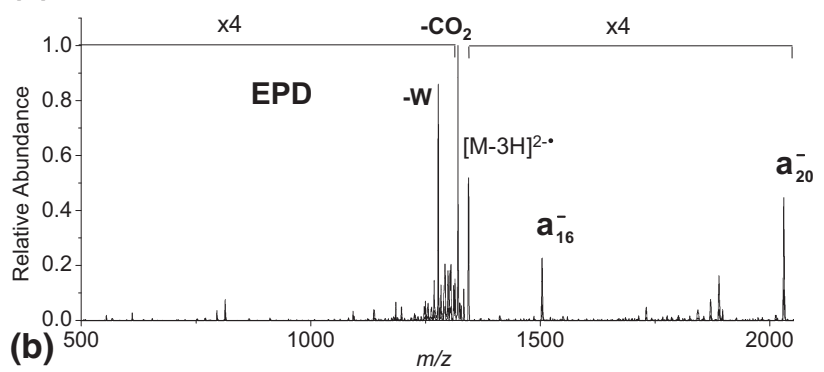

Figure 3. Tandem mass spectrometry of M2 TMP, G7@V7 peptide variant, performed on the $[\mathrm{M}-3 \mathrm{H}]^{3-}$ precursor ions (a) EDD FT-ICR mass spectrum. (b) EPD LTQ mass spectrum. Only the most intense fragments have been annotated on the spectra.

abundant. This result has to be contrasted with the results obtained for the acidic form of Substance P and is presumably due to the numerous acidic groups (Asp, Ser, and C-terminus) in the peptide. Thus, the acidic groups in M2 TMP and their variants can produce fragments that accommodate negative charges either on $\mathrm{N}$-terminal or on C-terminal sides. The observation of $z$ ions may be due to the presence of two acidic residues at the C-terminal side. Indeed the formation of fragments with a negative charge located at the C-terminal (e.g., $\mathrm{z}$ ions) may be favored.

In EPD of M2 TMP, G7@V7, intense losses of $\mathrm{CO}_{2}$ and tryptophan radical side-chain $(-\mathrm{W})$ molecules are observed, Figure $3 \mathrm{~b}$. Series of $a, x$, and $z$ product ions are observed due to the backbone cleavages, with a better sequence coverage than in EDD, Figure $4 \mathrm{~b}$. However, mainly $a$-ions are abundant although present in radical form and may be unstable. In particular, $a_{16}$ and $a_{20}$ ions have a high intensity compared with other a-ions. The $a_{20}$ fragment ions are due to the peptide backbone rupture at the C-terminal side of the tryptophan residue. As already discussed, the observation of an enhanced fragmentation close to the tryptophan residue in EPD may be due to the formation of neutral radical form of the tryptophan side chain (see Scheme $4 \mathbf{b})$. The important loss of $129 \mathrm{Da}(-\mathrm{W})$ observed by EPD is also in favor of the presence of a neutral tryptophan radical.

Interestingly, $a_{16}$ ions are also observed with a high intensity and correspond to fragments that are formed by peptide backbone cleavage at the C-terminal side of the histidine residue. Similarly to tryptophan, the ob- servation of an enhanced fragmentation close to the histidine residue may be due to preferential radical migration close to histidine (see Scheme 5). This is related to the recent observation that electron reduction of imidazolium ions forms peptide imidazolium radicals that can undergo backbone cleavage, side-chain loss, or isomerization [33]. Furthermore, iodination of tyrosine and histidine was recently used to generate tyrosine and histidine radical peptides in the gas phase by UV photo-cleavage of carbon-iodine bonds [34].

Furthermore, numerous $z$-ions are observed by EPD of M2 TMP, G7@V7 peptide, whereas these ions are absent in EDD. In particular, $z_{5}$ and $z_{9}$ ions have higher intensity compared with other $z$-ions. These ions (and their equivalent $c$-ions), in particular $c$ - and $z$-ions close to tryptophan residues, were also recently observed by EPD of melittin peptide dianions [21]. From a mechanistic point of view, the intense $c$ and $z$ fragment ions resulting from a cleavage in the vicinity of Trp could originate from the initial formation of a cationic radical
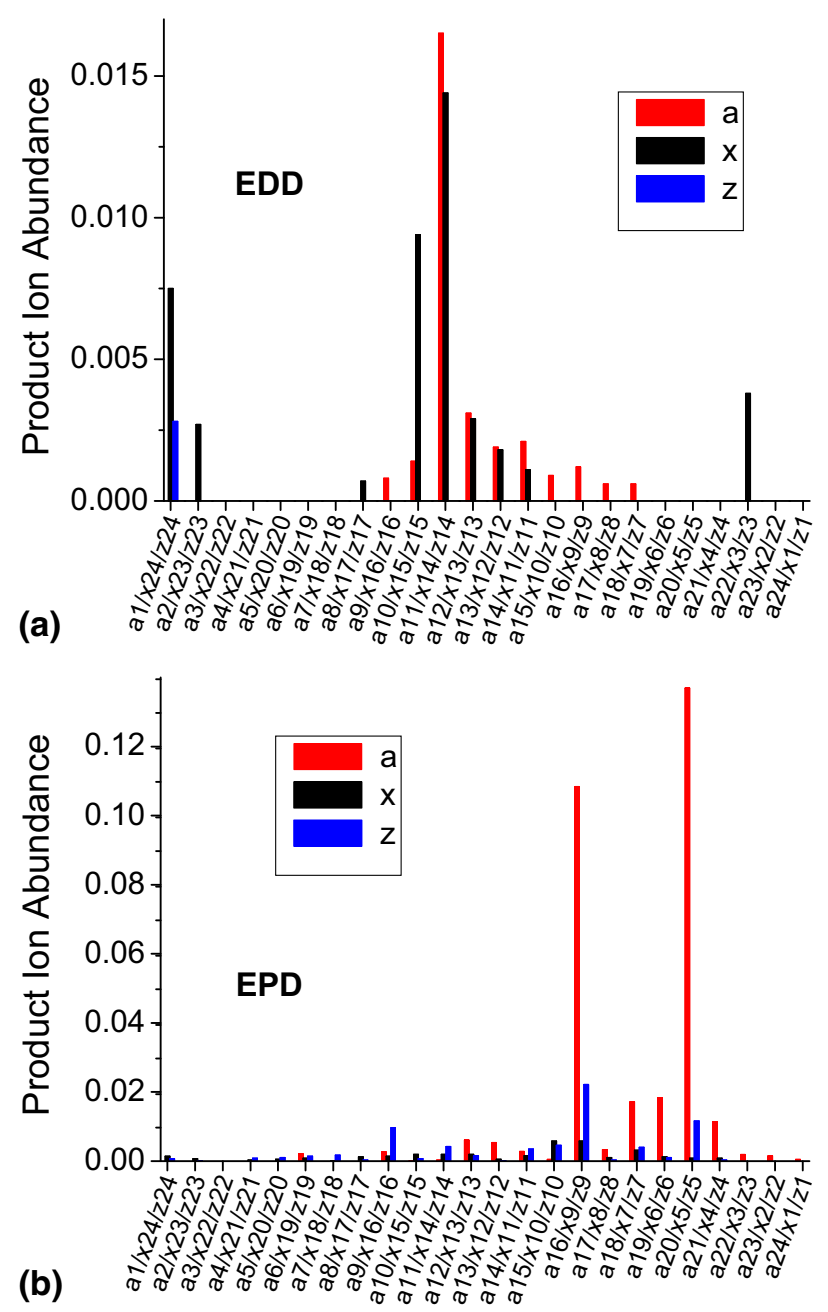

Figure 4. (a) Product ion abundance distribution ( $a, x$, and $z$ fragment intensities) obtained by EDD of triply deprotonated M2 TMP, G7@V7. (b) Product ion abundance distribution ( $a, x$, and $z$ fragment intensities) obtained by EPD of triply deprotonated M2 TMP, G7@V7. 

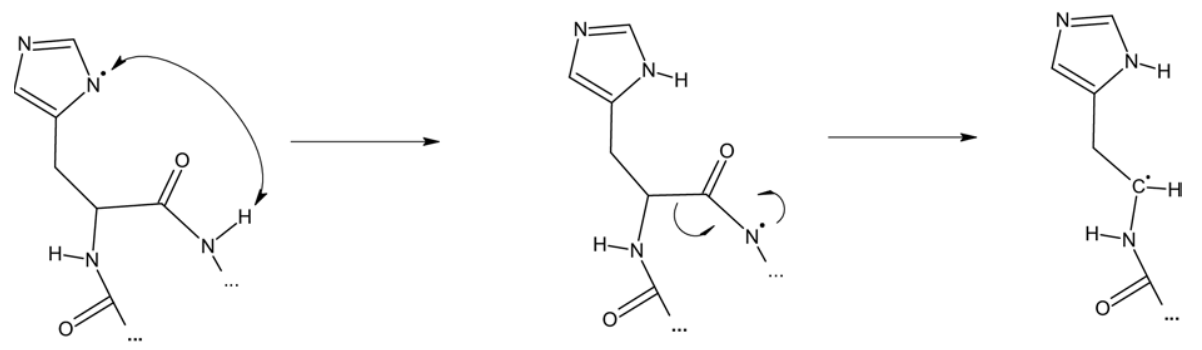

Scheme 5

on the indolic side group of tryptophan residue (due to photoionization of the indole group). In this case, electronic relaxation after photoexcitation (that occurs mainly on Trp at $260 \mathrm{~nm}$ ) would not lead to the loss of an electron from one of the negative groups of the peptide but to the ionization of the indolic residue, leading to the formation of a radical cation residue; $c$ and $z$ fragment formation around Trp were found to be particularly abundant for melittin peptide [21]. The competition between the ionization of the indole group or the electron loss from the negative sites may depend on the conformation. Subsequent favored recombination across the adjacent $\mathrm{N}-\mathrm{C}_{\alpha}$ bond would leave a neutral tryptophan (see Scheme 6).

Finally, Figure 5 displays the product ion abundance (PIA) distribution for 3 M2 TMP peptide variants and the wild type peptide obtained by EPD and EDD. First, PIA distributions for $a, x$, and $z$ ions obtained by EPD are very different from those obtained by EDD. The PIA distribution obtained by EPD is sequence-wide: $a, x$, and $z$ ions are observed all along the peptide sequences. As already discussed, the collisional activation step after radical formation and isolation may favor radical migration and account for the wide sequence coverage. The enhanced $a_{20}$ ion is observed for all the variants by EPD, confirming the key role of tryptophan in the observation of this fragment. Interestingly, the $a_{16}$ ion is observed for all the variants except the M2 TMP, G16@H16 (where the histidine residue has been re- placed by a glycine residue). These results also confirm the role of histidine in the observation of enhanced $a$ fragment ions close to histidine residues. The distribution of fragments for M2 TMP, G16@H16 is different from the one observed for the other variants. Presumably, incorporation of Gly at position 16 drastically affects the overall peptide conformation. As a result, more globular than extended $\alpha$-helical conformation can be adopted due to the additional, well-known, flexibility of the Gly residue. Therefore, charge solvation on the backbone amide nitrogens around $\mathrm{Gly}_{16}$ due to interaction with, for example, $\mathrm{Asp}_{3}, \mathrm{Asp}_{23}$, or Cterminal carboxyl, can be enhanced. Furthermore, the three most intense fragments considered $\left(x_{9}, x_{10}\right.$, and $z_{11}$ ) are due to the backbone rupture next to Leu residues, whereas Leu residues are known to provide additional radical stabilization compared with, for example, Ile. In this case, M2 TMP, G16@H16 adopts extended $\alpha$ helical conformation instead of a globular one; side-chain interaction of the Gly ${ }_{16}$ with the $\operatorname{Trp}_{20}$ located one $\alpha$-helical turn away may be responsible for radical migration to the vicinity of $\mathrm{Gly}_{16}$, its stabilization by $\mathrm{Leu}_{15}$ and $\mathrm{Leu}_{17}$, and subsequent backbone ruptures with further Leu-mediated product ion radical stabilization.

On the other hand, in EDD, the PIA distributions are more sensitive to the sequence of the M2 TMP variants. For the four peptides studied, the fragments observed for $a$ and $x$ ions are mainly localized near the center of

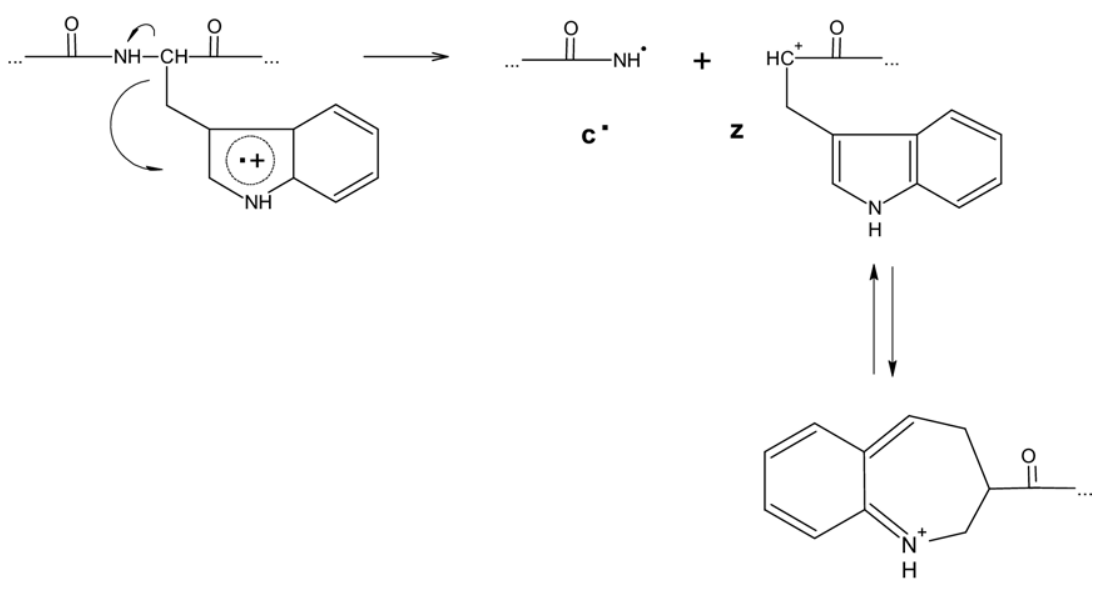

Scheme 6 

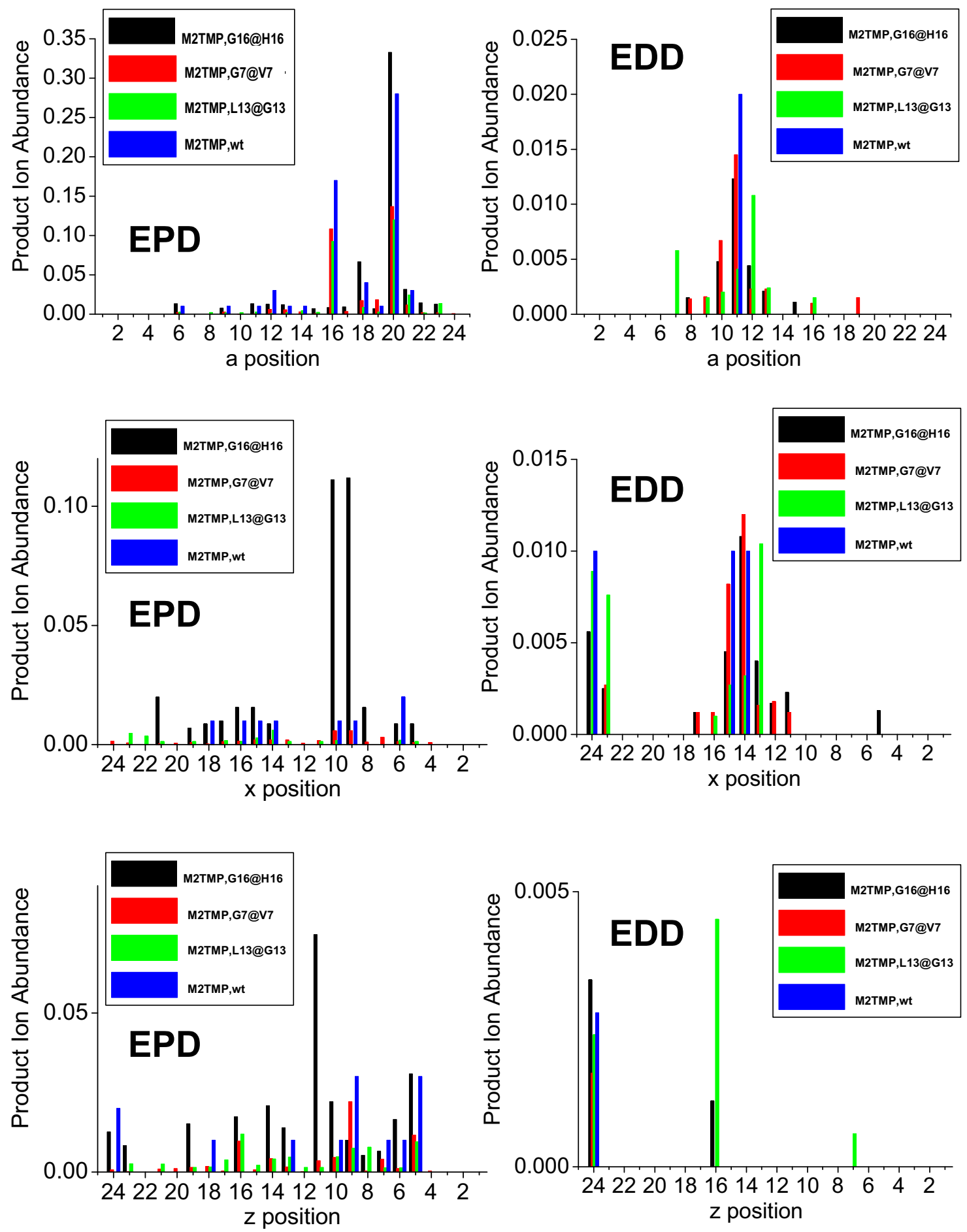

Figure 5. EPD and EDD product ion abundance distribution for M2 TMP peptide variants, (left): product ion abundance for $a, x$, and $z$ fragment ion types generated with EPD; (right): product ion abundance for $a, x$, and $z$ fragment ion types generated with EDD.

the peptide, specifically around $\mathrm{Ile}_{11}-\mathrm{Ile}_{12}$ for $a$-ions and $\mathrm{Ile}_{12}-\mathrm{Gly}_{13}\left(\mathrm{x}_{14}\right)$ for $x$-ions, and characterized by a sharp distribution. In EDD, energetic electron bombardment of precursor ions results in an electronic excitation and a rapid deposition of energy upon electron detachment thus reducing time for radical rearrangement compared with EPD. We can assume that the N- and C-terminally located negative charges solvate the backbone amide nitrogens in the middle of the peptide sequence, around the $\mathrm{Gly}_{13}$ residue that is known to induce a kink in the wild type M2 TMP structure [35, 36]. Furthermore, formation of backbone amide nitrogen radicals in the middle of the sequence would decrease the dissociation energy barrier for the backbone bonds in their vicinity. 
Therefore, conformation-dependent radical formation location can be used to rationalize the data shown in Figure 5. Without assuming these globular structures and interpreting the data for the possible extended $\alpha$-helical structures of M2 TMP variants may lead to the hypothesis of excitation energy relaxation along the peptide sequence and subsequent deposition in the area of the largest structural "defect" - a kink around Gly $_{13}$ [37].

\section{Conclusion}

In summary, we compared product-ion mass spectra produced by electron detachment dissociation (EDD) and electron photodetachment dissociation (EPD) of multi-deprotonated peptides. Both methods, EDD and EPD, involve the electron emission-induced formation of a radical oxidized species from a multi-deprotonated precursor peptide. While electron irradiation results in the formation of excited radical anionic species that fragment spontaneously, laser irradiation results in radical ionic species with low excess internal energy and additional vibrational activation of the oxidized species is necessary to yield fragment ions (activatedEPD). Product-ion mass spectra display mainly fragment ions resulting from backbone cleavages of $\mathrm{C}_{\alpha}-\mathrm{C}$ bond ruptures yielding $a$ and $x$ ions, but $c$ and $z$ ions may also be observed. Although EDD and EPD methods involve the generation of a charge-reduced radical anion intermediate by electron emission, the product ion abundance distributions are drastically different. This shows that the dissociation pathways are not only driven by the initial charge locations. EPD product ions are predominantly formed near tryptophan, tyrosine, and histidine residues, whereas in EDD the negative charge solvation sites on the backbone seem to be the most favorable for the nearby bond dissociation. This difference in product ions is attributed to the importance of radical migration in EPD. In activated-EPD, the collisional activation step after radical formation and isolation may favor radical migration. This would account for the wider sequence coverage than in EDD and yield intense fragment ions close to residues that are known to stabilize radicals. In the future, optical spectroscopy [30] could be used to probe the localization of radicals and confirm this hypothesis. The advantage of activation and radical migration for wide sequence coverage in EPD will have to be validated for a large panel of peptides. In EDD, energetic electron bombardment of precursor ions results in an electronic excitation and a rapid deposition of energy upon electron detachment, thus reducing time for radical rearrangement compared with EPD.

\section{Acknowledgments}

The authors thank Hisham Ben-Hamidane for discussions and for providing samples of M2 TMP peptide variants. They are grateful to Thermo Fisher Scientific, Bremen, Germany, for the access to the 7 T LTQ FTMS for EDD experiments. V.L., C.B., J.L., R.A., and P.D. acknowledge support from the CNRS, the Institut de Chimie de Lyon and EGIDE (PHC project 19153UH). Y.O.T. and A.V. acknowledge financial support from the Swiss National Science Foundation (SNF project 200021-125147/1) and Swiss Academy for Engineering Sciences (SATW project 2009-28).

\section{References}

1. Gevaert, K.; Vandekerckhove, J. Protein Identification Methods in Proteomics. Electrophoresis 2000, 21, 1145-1154.

2. Han, X. M.; Aslanian, A.; Yates, J. R. Mass Spectrometry for Proteomics. Curr. Opin. Chem. Biol. 2008, 12, 483-490.

3. Yates, J. R. Mass Spectrometry and the Age of the Proteome. J. Mass Spectrom. 1998, 33, 1-19.

4. Heck, A. J. R.; van den Heuvel, R. H. H. Investigation of Intact Protein Complexes by Mass Spectrometry. Mass Spectrom. Rev. 2004, 23, 368 389

5. Cooper, H. J.; Hakansson, K.; Marshall, A. G. The Role of Electron Capture Dissociation in Biomolecular Analysis. Mass Spectrom. Rev. 2005, 24, 201-222.

6. Zubarev, R. A. Reactions of Polypeptide Ions with Electrons in the Gas Phase. Mass Spectrom. Rev. 2003, 22, 57-77.

7. Laskin, J.; Futrell, J. H. Activation of Large Ions in FT-ICR Mass Spectrometry. Mass Spectrom. Rev. 2005, 24, 135-167.

8. Wells, J. M.; McLuckey, S. A. In Biological Mass Spectrometry, Vol. CDII Elsevier: San Diego, 2005; p. 148-185.

9. Dongre, A. R.; Jones, J. L.; Somogyi, A.; Wysocki, V. H. Influence of Peptide Composition, Gas-Phase Basicity, and Chemical Modification on Fragmentation Efficiency: Evidence for the Mobile Proton Model. J. Am. Soc. Mass Spectrom. 1996, 118, 8365-8374.

10. Zubarev, R. A.; Kelleher, N. L.; McLafferty, F. W. Electron Capture Dissociation of Multiply Charged Protein Cations. A Nonergodic Process. J. Am. Chem. Soc. 1998, 120, 3265-3266.

11. Mikesh, L. M.; Ueberheide, B.; Chi, A.; Coon, J. J.; Syka, J. E. P.; Shabanowitz, J.; Hunt, D. F. The Utility of ETD Mass Spectrometry in Proteomic Analysis. BBA-Proteins Proteom. 2006, 1764, 1811-1822.

12. Syka, J. E. P.; Coon, J. J.; Schroeder, M. J.; Shabanowitz, J.; Hunt, D. F. Peptide and Protein Sequence Analysis by Electron Transfer Dissociation Mass Spectrometry. Proc. Natl. Acad. Sci. U.S.A. 2004, 101, $9528-$ 9533.

13. Coon, J. J.; Shabanowitz, J.; Hunt, D. F.; Syka, J. E. P. Electron Transfer Dissociation of Peptide Anions. J. Am. Soc. Mass Spectrom. 2005, 16, $880-882$.

14. Kjeldsen, F.; Silivra, O. A.; Ivonin, I. A.; Haselmann, K. F.; Gorshkov, M. Zubarev, R. A. Calphabond C Backbone Fragmentation Dominates in Electron Detachment Dissociation of Gas-Phase Polypeptide Polyanions. Chem. Eur. J. 2005, 11, 1803-1812.

15. Budnik, B.; Haselmann, K.; Zubarev, R. Electron Detachment Dissociation of Peptide Dianions: An Electron-Hole Recombination Phenomenon. Chem. Phys. Lett. 2001, 342, 299-302.

16. Haselmann, K. F.; Budnik, B. A.; Kjeldsen, F.; Nielsen, M. L.; Olsen, J. V.; Zubarev, R. A. Electronic Excitation Gives Informative Fragmentation of Polypeptide Cations and Anions. Eur. J. Mass Spectrom. 2002, 8, 117-121.

17. Polfer, N.; Huzarska, M.; Kaplan, D.; Easterling, M. Proceedings of the 57th ASMS Conference on Mass Spectrometry and Allied Topics; Philadelphia, PA, May, 2009.

18. Anusiewicz, I.; Jasionowski, M.; Skurski, P.; Simons, J. Backbone and Side-Chain Cleavages in Electron Detachment Dissociation (EDD). J. Phys. Chem. A 2005, 109, 11332-11337.

19. Gabelica, V.; Tabarin, T.; Antoine, R.; Rosu, F.; Compagnon, I.; Broyer, M.; De Pauw, E.; Dugourd, P. Electron Photodetachment Dissociation of DNA Polyanions in a Quadrupole Ion Trap Mass Spectrometer. Anal. Chem. 2006, 78, 6564-6572.

20. Antoine, R.; Joly, L.; Tabarin, T.; Broyer, M.; Dugourd, P.; Lemoine, J. Photo-Induced Formation of Radical Anion Peptides. Electron Photodetachment Dissociation Experiments. Rapid Commun. Mass Spectrom. 2007, 21, 265-268.

21. Larraillet, V.; Antoine, R.; Dugourd, P.; Lemoine, J. Activated-Electron Photodetachment Dissociation for the Structural Characterization of Protein Polyanions. Anal. Chem. 2009, 81, 8410-8416.

22. Joly, L.; Antoine, R.; Broyer, M.; Lemoine, J.; Dugourd, P. Electron Photodetachment from Gas Phase Peptide Dianions. Relation with Optical Absorption Properties. J. Phys. Chem. A 2008, 112, 898-903.

23. Tsybin, Y. O.; Hendrickson, C. L.; Beu, S. C.; Marshall, A. G. Impact of Ion Magnetron Motion on Electron Capture Dissociation Fourier Transform Ion Cyclotron Resonance Mass Spectrometry. (Diethard Bohme Honor Issue). Int. J. Mass Spectrom 2006, 255/256, 144-149.

24. Matheis, K.; Joly, L.; Antoine, R.; Lepine, F.; Bordas, C.; Ehrler, O. T. Allouche, A.-R.; Kappes, M. M.; Dugourd, P. Photoelectron Spectroscopy of Gramicidin Polyanions: Competition Between Delayed and Direct Emission. J. Am. Chem. Soc. 2008, 130, 15903-15906.

25. Kweon, H. K.; Hakansson, K. Metal Oxide-Based Enrichment Combined with Gas-Phase Ion-Electron Reactions for Improved Mass Spectromet- 
ric Characterization of Protein Phosphorylation. J. Proteome Res. 2008, 7, 749-755.

26. Ben-Hamidane, H.; He, H.; Tsybin, O. Y.; Emmett, M. R.; Hendrickson, C. L.; Marshall, A. G.; Tsybin, Y. O. Periodic Sequence Distribution of Product Ion Abundances in Electron Capture Dissociation of Amphipathic Peptides and Proteins. J. Am. Soc. Mass Spectrom. 2009, 20, 1182-1192.

27. Joly, L.; Antoine, R.; Allouche, A. R.; Broyer, M.; Lemoine, J.; Dugourd, P. Optical Properties of Isolated Hormone Oxytocin Dianions: Ionization, Reduction, and Copper Complexation Effects. J. Phys. Chem. A 2009, 113, 6607-6611.

28. Joly, L.; Antoine, R.; Albrieux, F.; Ballivian, R.; Broyer, M.; Chirot, F.; Lemoine, J.; Dugourd, P.; Greco, C.; Mitric, R.; Bonacic-Koutecky, V. Optical and Structural Properties of Copper-Oxytocin Dications in the Gas Phase. J. Phys. Chem. B 2009, 113, 11293-11300.

29. Bowie, J. H.; Brinkworth, C. S.; Dua, S. Collision-Induced Fragmentations of the $(\mathrm{M}-\mathrm{H})^{-}$Parent Anions of Underivatized Peptides: An Aid to Structure Determination and Some Unusual Negative Ion Cleavages. Mass Spectrom. Rev. 2002, 21, 87-107.

30. Joly, L.; Antoine, R.; Allouche, A.-R.; Dugourd, P. Formation and Spectroscopy of a Tryptophan Radical Containing Peptide in the Gas Phase. J. Am. Chem. Soc. 2008, 130, 13832-13833.
31. Kovacs, F. A.; Denny, J. K.; Song, Z.; Quine, J. R.; Cross, T. A. Helix Tilt of the M2 Transmembrane Peptide from Influenza A Virus: An Intrinsic Property. J. Mol. Biol. 2000, 295, 117-125.

32. Tsybin, Y. O.; Ben-Hamidane, H.; Vorobyev, A.; Schmid, A.; Tsybin, O. Y.; Corminbeuf, C.; Pouthier, V. Proceedings of the 56th ASMS Conference on Mass Spectrometry and Allied Topics, Denver, June, 2008.

33. Turecek, F.; Yao, C.; Fung, Y. M. E.; Hayakawa, S.; Hashimoto, M.; Matsubara, H. Histidine-Containing Radicals in the Gas Phase. J. Phys. Chem. B 2009, 113, 7347-7366.

34. Liu, Z.; Julian, R. R. Deciphering the Peptide Iodination Code. J. Am. Soc. Mass Spectrom. 2009, 20, 965-971.

35. Yi, M.; Cross, T. A.; Zhou, H. X. Conformational Heterogeneity of the M2 Proton Channel and a Structural Model for Channel Activation. Proc. Natl. Acad. Sci. U.S.A. 2009, 106, 13311-13316.

36. Witter, R.; Nozirov, R.; Sternberg, U.; Cross, T. A.; Ulrich, A. S.; Fu, R. Solid-State 19F NMR Spectroscopy Reveals that trp41 Participates in the Gating Mechanism of the M2 Proton Channel of Influenza 1 Virus. J. Am. Chem. Soc 2008, 130, 918-924.

37. Pouthier, V.; Tsybin, Y. O. Amide-I Relaxation-Induced Hydrogen Bond Distortion: An Intermediate in Electron Capture Dissociation Mass Spectrometry of $\alpha$-helical Peptides? J. Chem. Phys. 2008, 129, 095106. 